\title{
Bone Metastasis in Breast Cancer
}

\author{
Ingo J. Diel \\ CGG Klinik GmbH, Schwerpunktpraxis Gynäkologische Onkologie Mannheim, Germany
}

The occurrence of bone metastases is a catastrophic event for breast cancer patients. Bone metastases are not only a sign of the incurability of the disease but also associated with typical complications which considerably limit the quality of life of the patient: bone pain, pathological fractures, and spinal cord compression syndromes, among others. Women with bone metastases nevertheless have long survival times, particularly if no visceral metastases are present. This means that within the course of the disease patient as well as therapist are confronted with new complications again and again. Besides the treatment of these complications their avoidance is the most important goal.

There is a broad armamentarium of local and systemic treatment options for bone metastases. It is of great importance that the therapist is familiar with all possibilities and choses the right treatment for the patient at the time. Chemoand endocrine therapies, antibodies, small molecules, osteoprotective substances (bisphosphonates and denosumab) are part of the systemic options. There are good national and international guidelines for all these types of therapy. As well, general guidelines for analgesic medication, bone-seeking therapy with radionuclides, and external radiotherapy are available, albeit not specifically for bone metastases from breast cancer. The assessment of surgical methods is far more difficult since, although all methods seem effective and necessary, the number of randomized studies is small and also not specific for the different tumor entities. At long last, the treatment of patients with bone metastases is a task for the 'arts of medicine'. This means that the success of treatment and the patient's quality of life is related to the experience of the physician and his readiness not to give up the patient.

The contributions in the focus on bone metastases in this issue of BREAST CARE represent a good overview of current and specific treatments which can be used against metastatic bone disease due to breast cancer: The overview of Gnant and colleagues [1] comprehensively describes the well-established treatment of osteoprotection with bisphosphonates and denosumab. For approximately 25 years bisphosphonates were widely used for the reduction of skeletal events and the prolongation of event-free intervals. In addition, these drugs improve the quality of life of the patients and reduce bone pain. Since August 2011 the first receptor activator of nuclear factor- $\kappa \mathrm{B}$ ligand (RANKL) antibody (denosumab) got the European approval for the treatment of skeletal events in metastatic bone disease. The therapeutical goal is identical with that of bisphosphonates (targeting the osteoclast), but the mode of action is different. Denosumab has shown superiority for the reduction of skeletal complications of metastatic bone disease compared with zoledronic acid.

The second contribution by Schneider, Voltz, and Gärtner [2] deals with the analgesic drug therapies for bone metastases. Until few years ago it was largely unknown that nerves reach also into the bone itself and not only into the periosteum and endosteum. The treatment principles aren't different from those for the treatment of pain from other metastases and tumors: we know that bone pain is a so-called mixedpain syndrome. That means different medications, including antiinflammatory and osteoprotective drugs, must be combined with opioids or morphines. The article closes with 11 very useful rules for the treatment of metastatic bone pain.

The following article is a survey of Fischer and Kampen [3] concerned with treatment options with bone-seeking substances (radioisotopes). While in the past most results were generated for patients with prostate cancers, there are now more and more new studies related to the outcome of breast cancer patients. The effectivity of radionuclide treatment is highest for osteoblastic metastases but an effect could also be shown for lytic or mixed lesions in breast cancer. In the future, more patients should be treated earlier, when the tumor burden is low and not only in the last stage of the disease. The method can possibly be used following an adjuvant therapy of breast cancer to destroy micrometastatic cell clusters.

The article by Feyer and Steingräber [4] presents radiotherapy as the standard of care of local therapy in symptomatic metastases. Treatment goals are clear: reduction of the tumor mass, improvement of bone pain and preservation or reconstruction of bone stability. Different schemes are

\section{KARGER}

Fax +497614520714

Information@Karger.de

www.karger.com
(C) 2012 S. Karger GmbH, Freiburg

$1661-3791 / 12 / 0072-0090 \$ 38.00 / 0$

Accessible online at:

www.karger.com/brc
Prof. Dr. med. Ingo Diel

Head of the German Osteooncological Society (DOG)

CGG Klinik GmbH, Schwerpunktpraxis Gynäkologische Onkologie Mannheim

Quadrat P 7, 16-18, 68161 Mannheim, Germany

Tel. +49 621 1250 64-20, Fax -39

idiel@spgo-mannheim.de 
described for the indication and fractionation of irradiation. In addition important questions are discussed related to the problems of secondary radiotherapy and to the treatment of asymptomatic metastases. To this day, we are not certain when radiotherapy should be started in mono- or oligometastatic disease. Finally the value of radiotherapy for spinal cord compression is described with or without decompression surgery.

Obviously, orthopedic and traumatological operations are also an important part in the management of patients with bone metastases. There is a variety of possibilities of improving the care of patients with bone metastases with actual or imminent threat of fractures. Treatment goals are pain reduction and restoration of stability and mobility. The multidisciplinary management, detailed indications, and technical procedures of surgical treatment of the axial and appendicular skeleton are complex. These methods are nowadays included in a comprehensive concept of the treatment of metastatic bone disease, but extend beyond the scope of this issue of BrEAST CARE. Since 'Surgical Treatment' is the main subject of issue $5 / 2012$ of this journal, the respective overview will be published there - demonstrating another important piece in our quest to put an end to the therapeutical nihilism in these far advanced disease situations.

The original article of Conde and colleagues [5] deals with the measurement of bone mineral density (BMD) in middleaged breast cancer survivors. Low BMD is not uncommon in this population, as breast cancer treatment, ovarian failure, and contraindication to hormone therapy may intensify bone loss in addition to factors that negatively influence BMD in healthy women. These findings may prove useful when establishing and implementing strategies to minimize bone loss in middle-aged breast cancer survivors.

To improve the treatment of patients with bone metastases, the German Osteooncological society (DOG) was founded in April 2010. In this scientific community doctors with different specializations cooperate. A complex complication, such as metastatic bone disease, can only treated in the best way if we are ready to exchange and to share experience and knowledge with our colleagues. I am convinced that the articles on hand will support this important goal.

\section{Disclosure Statement}

The author received honoraria for speeches and consultations from Amgen, Novartis, and Roche.

\section{References}

1 Gnant M, Balic M, Petru E, Raunik W, Singer CF, Steger GG, Watzke IM, Brodowicz T: Treatment of bone metastases in patients with advanced breast cancer. Breast Care 2012;7: DOI: 10.1159/000338650.

2 Schneider G, Voltz R, Gärtner J: Cancer pain management and bone metastases: an update for the clinician. Breast Care 2012;7: DOI: 10.1159/000338579.

3 Fischer M; Kampen WU: Radionuclide therapy of bone metastases. Breast Care 2012;7: DOI: $10.1159 / 000337634$

4 Feyer P, Steingräber M: Radiotherapy of bone metastasis in breast cancer patients - current approaches. Breast Care 2012;7: DOI: 10.1159/000338724.

5 Conde DM, Pinto-Neto AM, Costa-Paiva L, Martinez EZ: Low bone mineral density in middle-aged breast cancer survivors: prevalence and associated factors. Breast Care 2012;7: DOI: 10.1159/000337763. 